\title{
Four-loop cusp anomalous dimension in QED
}

\author{
Andrey Grozin \\ PRISMA Cluster of Excellence, Johannes Gutenberg University, \\ Staudingerweg 9, 55128 Mainz, Germany \\ Budker Institute of Nuclear Physics SB RAS, \\ Lavrentyev st. 11, Novosibirsk 630090, Russia \\ Novosibirsk State University, \\ Pirogova st. 2, Novosibirsk 630090, Russia \\ E-mail: A.G.Grozin@inp.nsk.su
}

ABstract: The 4-loop $C_{F}^{3} T_{F} n_{l}$ and 5-loop $C_{F}^{4} T_{F} n_{l}$ terms in the HQET field anomalous dimension $\gamma_{h}$ are calculated analytically (the 4-loop one agrees with the recent numerical result [1]). The 4-loop $C_{F}^{3} T_{F} n_{l}$ and 5-loop $C_{F}^{4} T_{F} n_{l}$ terms in the cusp anomalous dimension $\Gamma(\varphi)$ are calculated analytically, exactly in $\varphi$ (the $\varphi \rightarrow \infty$ asymptotics of the 4-loop one agrees with the recent numerical result [2]). Combining these results with the recent 4-loop $d_{F F} n_{l}$ contributions to $\gamma_{h}$ and to the small- $\varphi$ expansion of $\Gamma(\varphi)$ up to $\varphi^{4}$ [3], we now have the complete analytical 4-loop result for the Bloch-Nordsieck field anomalous dimension in QED, and the small- $\varphi$ expansion of the 4-loop QED cusp anomalous dimension up to $\varphi^{4}$.

KEYwords: Effective Field Theories, Perturbative QCD, Renormalization Group, Wilson, 't Hooft and Polyakov loops

ARXIV EPRINT: 1805.05050 


\section{Contents}

1 Introduction 1

2 HQET field anomalous dimension: the $C_{F}^{L-1} T_{F} n_{l} \alpha_{s}^{L}$ terms 2

3 QCD cusp anomalous dimension: the $C_{F}^{L-1} T_{F} n_{l} \alpha_{s}^{L}$ terms 5

$\begin{array}{lll}4 & \text { QED results } & 7\end{array}$

\section{Introduction}

QCD problems with a single heavy quark $Q$ having momentum $P=M_{Q} v+p$ (where $M_{Q}$ is the on-shell mass and $v$ is some vector with $v^{2}=1$ ) can be described by heavy quark effective theory (HQET) if characteristic heavy-quark residual momentum $p$, as well as characteristic gluon and light-quark momenta $k_{i}$, are $\ll M_{Q}$ (see, e.g., [4-6]). The heavy quark is described by the field

$$
h_{v 0}=Z_{h}^{1 / 2}\left(\alpha_{s}(\mu), a(\mu)\right) h_{v}(\mu),
$$

where we use the $\overline{\mathrm{MS}}$ scheme, and $Z_{h}$ is a minimal renormalization constant. We use the covariant gauge: $-\left(\partial_{\mu} A_{0}^{\mu a}\right)^{2} /\left(2 a_{0}\right)$ is added to the Lagrangian, the gauge-fixing parameter is renormalized by the same minimal constant as the gluon field: $a_{0}=Z_{A}\left(\alpha_{s}(\mu), a(\mu)\right) a(\mu)$. The HQET heavy-quark field anomalous dimension is defined as $\gamma_{h}=d \log Z_{h} / d \log \mu$. The $h_{v 0}$ coordinate-space propagator in the $v$ rest frame has the form

$$
S_{h}(x)=\delta^{(d-1)}(\vec{x}) \theta\left(x^{0}\right) W\left(x^{0}\right),
$$

where $W(t)$ is the straight Wilson line along $v$ of length $t$. The heavy-quark field is QCD and HQET are related by the matching coefficient $z$ [7]:

$$
Q_{0}=z_{0}^{1 / 2} h_{v 0}+\mathcal{O}\left(1 / M_{Q}\right), \quad Q(\mu)=z^{1 / 2}(\mu) h_{v}(\mu)+\mathcal{O}\left(1 / M_{Q}\right) .
$$

The HQET field anomalous dimension $\gamma_{h}$ is known up to three loops [8, 9]. In the first of these papers, it was obtained as a by-product of the three-loop calculation of the heavyquark field renormalization constant in the on-shell scheme $Z_{Q}^{\text {os }}$, from the requirement that the renormalized matching coefficient $z(\mu)$ (1.3) must be finite; in the second paper, it was confirmed by a direct HQET calculation. Several color structures of the 4-loop result are also known: $C_{F}\left(T_{F} n_{l}\right)^{3}$ [10] $\left(n_{l}\right.$ is the number of light flavors), $C_{F}^{2}\left(T_{F} n_{l}\right)^{2}[11,12]$, $C_{F} C_{A}\left(T_{F} n_{l}\right)^{2}$ [1] (from the analytical $Z_{Q}^{\text {os }}$ result [13] using the finiteness of $z(\mu)$ ), $d_{F F} n_{l}$ [3]. Here $C_{R}(R=F, A)$ are the standard quadratic Casimirs: $t_{R}^{a} t_{R}^{a}=C_{R} \mathbf{1}_{R}\left(t_{R}^{a}\right.$ are the generators in the representation $R, \mathbf{1}_{R}$ is the corresponding unit matrix); $\operatorname{Tr} t_{F}^{a} t_{F}^{b}=T_{F} \delta^{a b}$; 
$d_{F F}=d_{F}^{a b c d} d_{F}^{a b c d} / N_{c}$, where $d_{R}^{a b c d}=\operatorname{Tr} t_{R}^{(a} t_{R}^{b} t_{R}^{c} t_{R}^{d)}$ (the brackets mean symmetrization), and $N_{c}=\operatorname{Tr} \mathbf{1}_{F}$. The remaining terms are known numerically [1], from the numerical 4-loop $Z_{Q}^{\text {os }}$ using the finiteness of $z(\mu)$ (1.3). Here I calculate the $C_{F}^{L-1} T_{F} n_{l} \alpha_{s}^{L}$ terms up to $L=5$ analytically (section 2); the $L=4$ term agrees with the numerical result [1].

If the heavy-quark velocity is substantially changed (e.g., a weak decay into another heavy quark), we have HQET with 2 unrelated fields $h_{v}, h_{v^{\prime}}$. At the effective-theory level this is described by the current

$$
J_{0}=h_{v^{\prime} 0}^{+} h_{v 0}=Z_{J}\left(\alpha_{s}(\mu)\right) J(\mu) .
$$

The minimal renormalization constant $Z_{J}$ is gauge invariant (unlike $Z_{h}$ ) because the current $J_{0}$ is color singlet. The anomalous dimension of this current, also known as the cusp anomalous dimension, is defined as $\Gamma(\varphi)=d \log Z_{J} / d \log \mu$, where $\cosh \varphi=v \cdot v^{\prime}$.

The QCD cusp anomalous dimension $\Gamma(\varphi)$ is known up to three loops [11, 14]. At $\varphi \ll 1$ it is a regular series in $\varphi^{2}$. At $\varphi \gg 1$ it is $\Gamma_{l} \varphi+\mathcal{O}\left(\varphi^{0}\right)[15]$, where $\Gamma_{l}$ is the light-like cusp anomalous dimension. Several color structures of the 4-loop $\Gamma(\varphi)$ are also known: $C_{F}\left(T_{F} n_{l}\right)^{3}[16], C_{F}^{2}\left(T_{F} n_{l}\right)^{2}[11,12]$. The $d_{F F} n_{l}$ term is known at $\varphi \ll 1$ up to $\varphi^{4}[3] .{ }^{1}$ For the $\varphi \gg 1$ asymptotics (i. e. $\Gamma_{l}$ ), both $n_{l}^{2}$ terms are known from combining the $C_{F}^{2}\left(T_{F} n_{l}\right)^{2}$ result $[11,12]$ and the large- $N_{c} N_{c}^{2} n_{l}^{2}$ result [18]. Large- $N_{c}$ results for $\Gamma_{l}$ at $n_{l}^{1}[2,18]$ and $n_{l}^{0}[2,20]$ are also known analytically. Contributions of individual color structures of $\Gamma_{l}$ at $n_{l}^{1,0}$ are only known numerically [2]. Here I calculate the $C_{F}^{L-1} T_{F} n_{l} \alpha_{s}^{L}$ terms up to $L=5$ in $\Gamma(\varphi)$ analytically, as exact functions of $\varphi$ (section 3). In particular, I find their $\varphi \gg 1$ asymptotics; the analytical $L=4$ result agrees with the numerical one [2].

In QED without light lepton flavors $\left(n_{l}=0\right)$, as explained below, both $\gamma_{h}$ and $\Gamma(\varphi)$ are exactly given by the one-loop formulas. When $n_{l} \neq 0$, higher corrections appear. Combining the 4-loop $\gamma_{h}$ results for $C_{F}\left(T_{F} n_{l}\right)^{3}[10], C_{F}^{2}\left(T_{F} n_{l}\right)^{2}[11,12], C_{F}^{3} T_{F} n_{l}$ (section 2) and $d_{F F} n_{l}$ [3] structures, I obtain the complete analytical 4-loop result for the Bloch-Nordsieck field anomalous dimension $\gamma_{h}$ in QED (section 4). Combining the 4-loop $\Gamma(\varphi)$ full results for $C_{F}\left(T_{F} n_{l}\right)^{3}[16], C_{F}^{2}\left(T_{F} n_{l}\right)^{2}[11,12], C_{F}^{3} T_{F} n_{l}$ (section 3) structures with the $d_{F F} n_{l}$ term [3] (expansion up to $\varphi^{4}$ ), I obtain the expansion of the 4-loop QED $\Gamma(\varphi)$ up to $\varphi^{4}$ (section 4).

\section{HQET field anomalous dimension: the $C_{F}^{L-1} T_{F} n_{l} \alpha_{s}^{L}$ terms}

This is a QED problem. Due to exponentiation [21, 22], the coordinate-space propagator of the Bloch-Nordsieck field (i.e. the straight Wilson line $W$ ) is

$$
W=\exp \left(\sum w_{i}\right)
$$

where $w_{i}$ are single-web diagrams. Due to $C$ parity conservation in QED, webs have even numbers of legs (figure 1 ). In QED with $n_{l}=0$ there is only 1 single-web diagram: figure $1 \mathrm{a}$ with the free photon propagators. Therefore, $\log W$ is exactly 1-loop; the $\beta$ function is 0 , and hence $\gamma_{h}$ is also exactly 1-loop. At $n_{l}>0$ corrections to the photon propagator in figure 1a appear. Webs with 4 legs (figure 1b) first appear at 4 loops; they have been

\footnotetext{
${ }^{1}$ Such expansion was first used in [17] at 2 loops.
} 


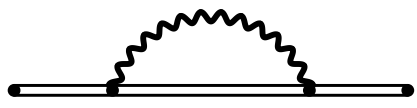

a

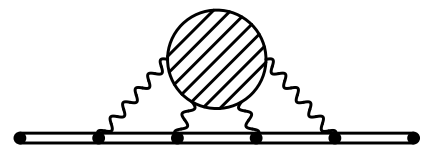

$\mathrm{b}$

Figure 1. Webs: (a) 2-leg (the thick line is the full photon propagator); (b) 4-leg (the blob is the sum of connected diagrams).

calculated in [3]. All contributions to $\log W(2.1)$ are gauge invariant except the 1-loop one, because proper vertex functions with any numbers of photon legs are gauge invariant and transverse with respect to each photon leg due to the QED Ward identities.

The full momentum-space photon propagator in the covariant gauge is

$$
D^{\mu \nu}(k)=-\frac{i}{k^{2}}\left(g^{\mu \nu}-\frac{k^{\mu} k^{\nu}}{k^{2}}\right) \frac{1}{1-\Pi\left(k^{2}\right)}-i a_{0} \frac{k_{\mu} k_{\nu}}{\left(k^{2}\right)^{2}},
$$

where $\Pi\left(k^{2}\right)$ is the photon self-energy:

$$
\Pi=\sum_{L=1}^{\infty} \Pi_{L} A_{0}^{L}\left(-k^{2}\right)^{-L \varepsilon}, \quad A_{0}=\frac{e_{0}^{2}}{(4 \pi)^{d / 2}} e^{-\gamma \varepsilon}
$$

( $e_{0}^{2}$ has dimensionality $m^{2 \varepsilon}$, so that the power of $-k^{2}$ is obvious; $\gamma$ is the Euler constant). Only the 0-loop term in (2.2) is gauge dependent. Writing $\Pi_{L}$ as $\tilde{\Pi}_{L} n_{l}+\left(n_{l}^{>1}\right.$ terms), we obtain in the Landau gauge $a_{0}=0$

$$
\begin{aligned}
& D^{\mu \nu}(k)=\tilde{D}_{0}^{\mu \nu}(k)+n_{l} \sum_{L=1}^{\infty} \tilde{\Pi}_{L} \tilde{D}_{L}^{\mu \nu}(k) A_{0}^{L}+\left(n_{l}^{>1} \text { terms }\right), \\
& \tilde{D}_{L}^{\mu \nu}(k)=\frac{i}{\left(-k^{2}\right)^{1+L \varepsilon}}\left(g^{\mu \nu}+\frac{k^{\mu} k^{\nu}}{-k^{2}}\right) .
\end{aligned}
$$

The $\overline{\mathrm{MS}}$ charge renormalization is

$$
\begin{aligned}
A_{0} & =\mu^{2 \varepsilon} \frac{\alpha(\mu)}{4 \pi} Z_{\alpha}(\alpha(\mu)), \\
\frac{d \log \alpha(\mu)}{d \log \mu} & =-2 \varepsilon-2 \beta(\alpha(\mu)), \quad \beta(\alpha)=\frac{1}{2} \frac{d \log Z_{\alpha}}{d \log \mu}=\sum_{L=1}^{\infty} \beta_{L}\left(\frac{\alpha}{4 \pi}\right)^{L}
\end{aligned}
$$

(note that here we call the $L$-loop $\beta$ function coefficient $\beta_{L}$, not $\beta_{L-1}$ as usually done; this makes subsequent formulas more logical). In QED $\log \left(1-\Pi\left(k^{2}\right)\right)=\log Z_{\alpha}+$ (finite); writing $\beta_{L}=\bar{\beta}_{L} n_{l}+\left(n_{l}^{>1}\right.$ terms $)$, we see that $1 / \varepsilon$ terms in $\tilde{\Pi}_{L}$ are related to $\bar{\beta}_{L}$ :

$$
\tilde{\Pi}_{L}=\frac{\bar{\beta}_{L}}{L} \frac{1}{\varepsilon}+\bar{\Pi}_{L}+\mathcal{O}(\varepsilon) .
$$

Here the $\beta$ function coefficients are [23]

$$
\bar{\beta}_{1}=-\frac{4}{3}, \quad \bar{\beta}_{2}=-4, \quad \bar{\beta}_{3}=2, \quad \bar{\beta}_{4}=46,
$$


and $[24]$

$$
\begin{aligned}
& \bar{\Pi}_{1}=-\frac{20}{9}, \quad \bar{\Pi}_{2}=16 \zeta_{3}-\frac{55}{3}, \quad \bar{\Pi}_{3}=-2\left(80 \zeta_{5}-\frac{148}{3} \zeta_{3}-\frac{143}{9}\right), \\
& \bar{\Pi}_{4}=2240 \zeta_{7}-1960 \zeta_{5}-104 \zeta_{3}+\frac{31}{3} .
\end{aligned}
$$

The coordinate-space full photon propagator is the Fourier transform of (2.4):

$$
\begin{aligned}
D^{\mu \nu}(x)= & \frac{1}{(4 \pi)^{d / 2}}\left[\bar{D}_{0}^{\mu \nu}(x)+n_{l} \sum_{L=1}^{\infty} \tilde{\Pi}_{L} \bar{D}_{L}^{\mu \nu}(x) A_{0}^{L}\right]+\left(n_{l}^{>1} \text { terms }\right), \\
\bar{D}_{L}^{\mu \nu}(x)= & \frac{\Gamma(1-(L+1) \varepsilon)}{\Gamma(1+L \varepsilon)}\left(\frac{4}{-x^{2}}\right)^{1-(L+1) \varepsilon} \\
& \times\left[-g^{\mu \nu}+\frac{g^{\mu \nu}+2(1-(L+1) \varepsilon) x^{\mu} x^{\nu} /\left(-x^{2}\right)}{2(1+L \varepsilon)}\right] .
\end{aligned}
$$

The sum of single-web diagrams (figure 1) in the Landau gauge, analytically continued to Euclidean $t=-i \tau$, is

$$
\log W=S_{1} A+n_{l} \sum_{L=2}^{\infty} S_{L} \tilde{\Pi}_{L-1} A^{L}+\left(n_{l}^{>1} \text { terms }\right)+\left(w_{>2 \text { legs }} \text { terms }\right), \quad A=A_{0}\left(\frac{\tau}{2}\right)^{2 \varepsilon} e^{2 \gamma \varepsilon},
$$

where the 1-loop HQET integral

$$
S_{L}=\frac{3-2 \varepsilon}{L \varepsilon(1-2 L \varepsilon)(1+(L-1) \varepsilon)} \frac{\Gamma(1-L \varepsilon)}{\Gamma(1+(L-1) \varepsilon)} e^{-(2 L-1) \gamma \varepsilon}=\frac{3}{L \varepsilon}+3+\frac{1}{L}+\mathcal{O}(\varepsilon)
$$

can be calculated in coordinate space (2.9), or as a Fourier transform of the momentumspace HQET propagator. Now we re-express $\log W$ via the renormalized $\alpha(\mu)$ at $\mu_{0}=$ $2 e^{-\gamma} / \tau$ (it is sufficient to do this in the 1-loop term) and obtain

$$
\begin{aligned}
\log W= & S_{1} \frac{\alpha}{4 \pi}+n_{l} \sum_{L=2}^{\infty}\left[S_{L}\left(\frac{\bar{\beta}_{L-1}}{L-1} \frac{1}{\varepsilon}+\bar{\Pi}_{L-1}\right)-S_{1} \frac{\bar{\beta}_{L-1}}{L-1} \frac{1}{\varepsilon}\right]\left(\frac{\alpha}{4 \pi}\right)^{L} \\
& +\left(n_{l}^{>1} \text { terms }\right)+\left(w_{>2 \text { legs }} \text { terms }\right)=\log Z_{h}+(\text { finite }) .
\end{aligned}
$$

Extracting $\log Z_{h}$ and differentiating it in $\log \mu$, we obtain $\gamma_{h}$. Restoring the color factors and adding the gauge dependent term ${ }^{2}$ we obtain

$$
\begin{aligned}
\gamma_{h} & =\frac{\alpha_{s}}{4 \pi}\left[2(a-3) C_{F}+T_{F} n_{l} \sum_{L=1}^{\infty}\left(-6 \bar{\Pi}_{L}+2 \bar{\beta}_{L}\right)\left(C_{F} \frac{\alpha_{s}}{4 \pi}\right)^{L}\right]+(\text { other color structures }) \\
& =2(a-3) C_{F} \frac{\alpha_{s}}{4 \pi}+T_{F} n_{l} C_{F}\left(\frac{\alpha_{s}}{4 \pi}\right)^{2}\left[\frac{32}{3}-6\left(16 \zeta_{3}-17\right) C_{F} \frac{\alpha_{s}}{4 \pi}\right.
\end{aligned}
$$

\footnotetext{
${ }^{2}$ In the arbitrary covariant gauge the extra term to be added to $w_{1}(2.10)$ is $\Gamma(-\varepsilon) e^{-\gamma \varepsilon} a_{0} A=$ $\Gamma(-\varepsilon) e^{-\gamma \varepsilon} a\left(\mu_{0}\right) \alpha\left(\mu_{0}\right) /(4 \pi)$, because in QED $Z_{\alpha}=Z_{A}^{-1}$. Hence the extra term to be added to $\log Z_{h}$ is purely 1-loop: $-(a / \varepsilon) \alpha /(4 \pi)$. In QED $d \log (a(\mu) \alpha(\mu)) / d \log \mu=-2 \varepsilon$ exactly, and hence the extra term in $\gamma_{h}(2.13)$ is also purely 1-loop: $2 a \alpha /(4 \pi)$.
} 


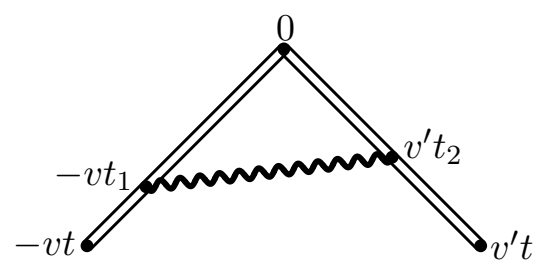

Figure 2. Cusp: the 2-leg web.

$$
\begin{aligned}
& +\frac{16}{3}\left(180 \zeta_{5}-111 \zeta_{3}-35\right)\left(C_{F} \frac{\alpha_{s}}{4 \pi}\right)^{2} \\
& \left.-6\left(2240 \zeta_{7}-1960 \zeta_{5}-104 \zeta_{3}-5\right)\left(C_{F} \frac{\alpha_{s}}{4 \pi}\right)^{3}+\mathcal{O}\left(\alpha_{s}^{4}\right)\right] \\
& + \text { (other color structures) }
\end{aligned}
$$

We have reproduced the $C_{F}^{2} T_{F} n_{l}$ term in the 3-loop anomalous dimension [8,9] by a simpler method. The coefficient of $C_{F}^{3} T_{F} n_{l}\left(\alpha_{s} / \pi\right)^{4}$ in $\frac{1}{2} \gamma_{h}$ is

$$
\frac{180 \zeta_{5}-111 \zeta_{3}-35}{96} \approx 0.189778
$$

in perfect agreement with the numerical result $0.1894 \pm 0.0030$ (table III in [1]).

\section{QCD cusp anomalous dimension: the $C_{F}^{L-1} T_{F} n_{l} \alpha_{s}^{L}$ terms}

Now we consider the Green function $<T\left\{h_{v}^{+}(x) J(0) h_{v^{\prime}}\left(x^{\prime}\right)\right\}>$. Up to obvious $\delta$ functions similar to (1.2), it is the broken Wilson line $W(\varphi)$ from $x=-v t$ to 0 and then to $x^{\prime}=v^{\prime} t^{\prime}$. Renormalization constants cannot depend on kinematics of Green functions we choose to calculate, and so we choose $t^{\prime}=t$ to have a single-scale problem. We have

$$
\log \frac{W(\varphi)}{W(0)}=\exp \left(\sum\left(w_{i}(\varphi)-w_{i}(0)\right)\right)
$$

where the sum runs over all single-web diagrams. Diagrams in which all photon vertices are to the left (or to the right) of the $J$ vertex cancel in $w_{i}(\varphi)-w_{i}(0)$. The remaining 2-leg webs are shown in figure 2. At 4 loops 4 -leg webs appear; they have been calculated $($ at $\varphi \ll 1)$ in $[3]$.

The the $L$-loop $n_{l}^{1}$ contribution is $(L \geq 2)$

$$
w_{L}(\varphi)=-\tilde{\Pi}_{L-1} n_{l} A_{0}^{L} e^{L \gamma \varepsilon} \int_{0}^{t} d t_{1} \int_{0}^{t} d t_{2} v_{\mu} v_{\nu}^{\prime} \bar{D}_{L-1}^{\mu \nu}\left(v t_{1}+v^{\prime} t_{2}\right)
$$

where $\bar{D}_{L}^{\mu \nu}(x)$ is given by (2.9). We can write it, together with the 1-loop Landau-gauge contribution, in the form

$$
w_{1}(\varphi)=V_{1}(\varphi) A, \quad w_{L}(\varphi)=V_{L}(\varphi) \tilde{\Pi}_{L-1} n_{l} A^{L},
$$

where

$$
V_{L}(\varphi)=4 \frac{\Gamma(1-u)}{\Gamma(1+u-\varepsilon)} e^{-(2 L-1) \gamma \varepsilon}\left[-I_{1}(\varphi) \cosh \varphi+\frac{u I_{1}(\varphi) \cosh \varphi-(1-u) I_{2}(\varphi)}{2(1+u-\varepsilon)}\right]
$$


$u=L \varepsilon$. The integrals $I_{1,2}(\varphi)$ are

$$
\begin{aligned}
I_{1}(\varphi) & =\int_{0}^{1} d t_{1} \int_{0}^{1} d t_{2}\left(e^{\varphi / 2} t_{1}+e^{-\varphi / 2} t_{2}\right)^{-1+u}\left(e^{-\varphi / 2} t_{1}+e^{\varphi / 2} t_{2}\right)^{-1+u} \\
& =\frac{e^{-2 u \varphi}}{4 u^{2} \sinh \varphi}\left(g_{1}(\varphi)-g_{2}(\varphi)\right), \\
I_{2}(\varphi) & =\int_{0}^{1} d t_{1} \int_{0}^{1} d t_{2}\left(e^{\varphi / 2} t_{1}+e^{-\varphi / 2} t_{2}\right)^{u}\left(e^{-\varphi / 2} t_{1}+e^{\varphi / 2} t_{2}\right)^{-2+u} \\
& =\frac{1}{2 u(1-u)}\left[1+\frac{e^{-2 u \varphi}}{2 \sinh \varphi}\left(e^{-\varphi} g_{1}(\varphi)-e^{\varphi} g_{2}(\varphi)\right)\right], \\
I_{1}(0) & =I_{2}(0)=\frac{2-2^{2 u}}{2 u(1-2 u)}, \\
g_{1}(\varphi) & =\left(e^{\varphi}+1\right)^{2 u} f_{1}\left(1-e^{\varphi}\right)-f_{1}\left(1-e^{2 \varphi}\right), \quad g_{2}(\varphi)=\left(e^{\varphi}+1\right)^{2 u} f_{2}\left(1-e^{\varphi}\right)-f_{2}\left(1-e^{2 \varphi}\right), \\
f_{1}(x) & ={ }_{2} F_{1}\left(\begin{array}{c}
-2 u,-u \\
1-2 u
\end{array}\right)=1+2 \operatorname{Li}_{2}(x) u^{2}+\mathcal{O}\left(u^{3}\right), \\
f_{2}(x) & ={ }_{2} F_{1}\left(\begin{array}{c}
-2 u, 1-u \\
1-2 u
\end{array}\right)=1+2 \log (1-x) u+\left(\log ^{2}(1-x)-2 \operatorname{Li}_{2}(x)\right) u^{2}+\mathcal{O}\left(u^{3}\right) .
\end{aligned}
$$

We obtain

$$
\begin{aligned}
V_{L}(\varphi)-V_{L}(0)= & -2 \frac{\varphi \operatorname{coth} \varphi-1}{L \varepsilon}+\bar{V}(\varphi)+\mathcal{O}(\varepsilon), \\
\bar{V}(\varphi)= & \operatorname{coth} \varphi\left[4 \operatorname{Li}_{2}\left(1-e^{2 \varphi}\right)-4 \operatorname{Li}_{2}\left(1-e^{\varphi}\right)+\varphi\left(4 \log \left(e^{\varphi}+1\right)+\varphi\right)\right] \\
& +2 \log \left(e^{\varphi}+1\right)-\varphi-6 \log 2+4=\bar{V}(-\varphi) .
\end{aligned}
$$

Similarly to (2.12), we substitute (3.10) into

$$
\log \frac{W(\varphi)}{W(0)}=\log Z_{J}+\text { (finite) }
$$

and re-express it via $\alpha\left(\mu_{0}\right)$ (it is sufficient to do this in the 1-loop term). Note that $\bar{V}(\varphi)$ does not depend on $L$; as a result, terms $\bar{\beta}_{L-1} \bar{V}(\varphi)$ cancel in $\log Z_{J}$ (in contrast to the first line of (2.13) where they contributed because of the $1 / L$ term in (2.11)). Differentiating $\log Z_{J}$ we obtain

$$
\begin{aligned}
\Gamma(\varphi)=4(\varphi \operatorname{coth} \varphi-1) \frac{\alpha_{s}}{4 \pi}\left[C_{F}\right. & \left.+T_{F} n_{l} \sum_{L=1}^{\infty} \bar{\Pi}_{L}\left(C_{F} \frac{\alpha_{s}}{4 \pi}\right)^{L}\right]+(\text { other color structures }) \\
=4(\varphi \operatorname{coth} \varphi-1) C_{F} \frac{\alpha_{s}}{4 \pi}\{ & 1+T_{F} n_{l} \frac{\alpha_{s}}{4 \pi}\left[-\frac{20}{9}+\left(16 \zeta_{3}-\frac{55}{3}\right) C_{F} \frac{\alpha_{s}}{4 \pi}\right. \\
& -2\left(80 \zeta_{5}-\frac{148}{3} \zeta_{3}-\frac{143}{9}\right)\left(C_{F} \frac{\alpha_{s}}{4 \pi}\right)^{2} \\
& \left.\left.+\left(2240 \zeta_{7}-1960 \zeta_{5}-104 \zeta_{3}+\frac{31}{3}\right)\left(C_{F} \frac{\alpha_{s}}{4 \pi}\right)^{3}+\mathcal{O}\left(\alpha_{s}^{4}\right)\right]\right\}
\end{aligned}
$$

+ (other color structures). 
Thus we have reproduced the 3-loop $C_{F}^{2} T_{F} n_{l}$ term in $[11,14]$. The coefficient of $2 T_{F} n_{l} C_{F}^{3}\left(\alpha_{s} /(4 \pi)\right)^{4}$ in the light-like cusp anomalous dimension $\Gamma_{l}$ is

$$
-4\left(80 \zeta_{5}-\frac{148}{3} \zeta_{3}-\frac{143}{9}\right) \approx-31.055431
$$

in perfect agreement with the numerical result $-31.00 \pm 0.4$ (table 2 in [2]).

\section{QED results}

The 4-loop anomalous dimension of the QED Bloch-Nordsieck field is now known completely analytically. Adding terms with higher powers of $n_{l}$ from $[11,12]$ and the 4-loop contribution of the webs with 4 legs [3], we obtain

$$
\begin{aligned}
\gamma_{h}= & 2(a-3) \frac{\alpha}{4 \pi}+\frac{32}{3} n_{l}\left(\frac{\alpha}{4 \pi}\right)^{2}+\left[-6\left(16 \zeta_{3}-17\right)+\frac{160}{27} n_{l}\right] n_{l}\left(\frac{\alpha}{4 \pi}\right)^{3} \\
+ & {\left[16\left(40 \zeta_{5}+\frac{32}{3} \pi^{2} \zeta_{3}-21 \zeta_{3}-\frac{32}{3} \pi^{2}-\frac{35}{3}\right)\right.} \\
& \left.-32\left(\frac{\pi^{4}}{15}-12 \zeta_{3}+\frac{103}{27}\right) n_{l}-\frac{256}{9}\left(\zeta_{3}-\frac{1}{3}\right) n_{l}^{2}\right] n_{l}\left(\frac{\alpha}{4 \pi}\right)^{4} .
\end{aligned}
$$

Adding terms with higher powers of $n_{l}[11,12]$ and the 4-legs webs contribution [3] (known only up to $\varphi^{4}$ ) to (3.11), we obtain the QED cusp anomalous dimension up to 4 loops

$$
\begin{aligned}
\Gamma(\varphi)=4(\varphi \operatorname{coth} \varphi-1) \frac{\alpha}{4 \pi}\left\{1+n_{l} \frac{\alpha}{4 \pi}\left[-\frac{20}{9}+\left(16 \zeta_{3}-\frac{55}{3}-\frac{16}{27} n_{l}\right) \frac{\alpha}{4 \pi}\right.\right. \\
\left.\left.-\frac{8}{9}\left(\frac{2}{5} \pi^{4}-80 \zeta_{3}+\frac{299}{9}-\frac{8}{3}\left(2 \zeta_{3}-\frac{1}{3}\right) n_{l}\right) n_{l}\left(\frac{\alpha}{4 \pi}\right)^{2}\right]\right\} \\
-\frac{8}{3} \varphi^{2}\left[80 \zeta_{5}+\frac{128}{3} \pi^{2} \zeta_{3}-\frac{40}{9} \pi^{4}-\frac{148}{3} \zeta_{3}-\frac{80}{9} \pi^{2}-\frac{143}{9}\right. \\
+\left(112 \zeta_{5}+\frac{512}{75} \pi^{2} \zeta_{3}-\frac{392}{225} \pi^{4}-\frac{6076}{75} \zeta_{3}+\frac{1256}{225} \pi^{2}+\frac{2371}{225}\right) \varphi^{2} \\
\left.+\mathcal{O}\left(\varphi^{4}\right)\right] n_{l}\left(\frac{\alpha}{4 \pi}\right)^{4} .
\end{aligned}
$$

The $n_{l} \alpha^{4}$ term is known only up to $\varphi^{4}$;

$$
\varphi \operatorname{coth} \varphi-1=\frac{\varphi^{2}}{3}\left(1-\frac{\varphi^{2}}{15}+\mathcal{O}\left(\varphi^{4}\right)\right)
$$

\section{Acknowledgments}

I am grateful to J. M. Henn and M. Stahlhofen for hospitality in Mainz and useful discussions; to M. Steinhauser and P. Marquard for discussing [1]; to A. Vogt for discussing [2]. The work has been supported by the PRISMA cluster of excellence, JGU Mainz, and partially by the Russian Ministry of Education and Science. 
Open Access. This article is distributed under the terms of the Creative Commons Attribution License (CC-BY 4.0), which permits any use, distribution and reproduction in any medium, provided the original author(s) and source are credited.

\section{References}

[1] P. Marquard, A.V. Smirnov, V.A. Smirnov and M. Steinhauser, Four-loop wave function renormalization in QCD and QED, Phys. Rev. D 97 (2018) 054032 [arXiv:1801.08292] [INSPIRE].

[2] S. Moch, B. Ruijl, T. Ueda, J.A.M. Vermaseren and A. Vogt, Four-loop non-singlet splitting functions in the planar limit and beyond, JHEP 10 (2017) 041 [arXiv:1707.08315] [INSPIRE].

[3] A. Grozin, J. Henn and M. Stahlhofen, On the Casimir scaling violation in the cusp anomalous dimension at small angle, JHEP 10 (2017) 052 [arXiv: 1708.01221] [INSPIRE].

[4] A.V. Manohar and M.B. Wise, Heavy quark physics, Camb. Monogr. Part. Phys. Nucl. Phys. Cosmol. 10 (2000) 1 [INSPIRE].

[5] A.G. Grozin, Heavy quark effective theory, Springer Tracts Mod. Phys. 201 (2004) 1 [INSPIRE].

[6] A.G. Grozin, Introduction to effective field theories. 3. Bloch-Nordsieck effective theory, HQET, arXiv: 1305.4245 [INSPIRE].

[7] A.G. Grozin, Matching heavy-quark fields in QCD and HQET at three loops, Phys. Lett. B 692 (2010) 161 [arXiv: 1004.2662] [INSPIRE].

[8] K. Melnikov and T. van Ritbergen, The three loop on-shell renormalization of $Q C D$ and QED, Nucl. Phys. B 591 (2000) 515 [hep-ph/0005131] [inSPIRE].

[9] K.G. Chetyrkin and A.G. Grozin, Three-loop anomalous dimension of the heavy-light quark current in HQET, Nucl. Phys. B 666 (2003) 289 [hep-ph/0303113] [INSPIRE].

[10] D.J. Broadhurst and A.G. Grozin, Matching QCD and HQET heavy-light currents at two loops and beyond, Phys. Rev. D 52 (1995) 4082 [hep-ph/9410240] [INSPIRE].

[11] A. Grozin, J.M. Henn, G.P. Korchemsky and P. Marquard, The three-loop cusp anomalous dimension in QCD and its supersymmetric extensions, JHEP 01 (2016) 140 [arXiv: 1510.07803] [INSPIRE].

[12] A. Grozin, Leading and next-to-leading large- $n_{f}$ terms in the cusp anomalous dimension and quark-antiquark potential, PoS (LL2016) 053 [arXiv: 1605.03886] [INSPIRE].

[13] R. Lee, P. Marquard, A.V. Smirnov, V.A. Smirnov and M. Steinhauser, Four-loop corrections with two closed fermion loops to fermion self energies and the lepton anomalous magnetic moment, JHEP 03 (2013) 162 [arXiv:1301.6481] [INSPIRE].

[14] A. Grozin, J.M. Henn, G.P. Korchemsky and P. Marquard, Three-loop cusp anomalous dimension in QCD, Phys. Rev. Lett. 114 (2015) 062006 [arXiv:1409.0023] [INSPIRE].

[15] G.P. Korchemsky and A.V. Radyushkin, Renormalization of the Wilson loops beyond the leading order, Nucl. Phys. B 283 (1987) 342 [InSPIRE].

[16] M. Beneke and V.M. Braun, Power corrections and renormalons in Drell-Yan production, Nucl. Phys. B 454 (1995) 253 [hep-ph/9506452] [INSPIRE]. 
[17] E. Bagan and P. Gosdzinsky, Two-loop renormalization scale dependence of the Isgur-Wise function, Phys. Lett. B 305 (1993) 157 [INSPIRE].

[18] J.M. Henn, A.V. Smirnov, V.A. Smirnov and M. Steinhauser, A planar four-loop form factor and cusp anomalous dimension in QCD, JHEP 05 (2016) 066 [arXiv:1604.03126] [INSPIRE].

[19] J. Davies, A. Vogt, B. Ruijl, T. Ueda and J.A.M. Vermaseren, Large- $n_{f}$ contributions to the four-loop splitting functions in QCD, Nucl. Phys. B 915 (2017) 335 [arXiv:1610.07477] [INSPIRE].

[20] J. Henn, R.N. Lee, A.V. Smirnov, V.A. Smirnov and M. Steinhauser, Four-loop photon quark form factor and cusp anomalous dimension in the large- $N_{c}$ limit of QCD, JHEP 03 (2017) 139 [arXiv: 1612.04389] [INSPIRE].

[21] J.G.M. Gatheral, Exponentiation of eikonal cross-sections in non-Abelian gauge theories, Phys. Lett. B 133 (1983) 90 [InSPIRE].

[22] J. Frenkel and J.C. Taylor, Non-Abelian eikonal exponentiation, Nucl. Phys. B 246 (1984) 231 [INSPIRE].

[23] S.G. Gorishny, A.L. Kataev, S.A. Larin and L.R. Surguladze, The analytical four-loop corrections to the QED $\beta$-function in the $M S$ scheme and to the QED $\psi$-function: total reevaluation, Phys. Lett. B 256 (1991) 81 [INSPIRE].

[24] B. Ruijl, T. Ueda, J.A.M. Vermaseren and A. Vogt, Four-loop QCD propagators and vertices with one vanishing external momentum, JHEP 06 (2017) 040 [arXiv:1703.08532] [INSPIRE]. 